\title{
Exit strategy of COVID-19 crisis and pathways of Vaccination
}

\author{
Indrani Roy ${ }^{1}$ \\ ${ }^{1}$ University College London * Correspondence
}

January 14, 2022

\begin{abstract}
There is a strong coordinated effort by vaccination groups all over the world to put an end to the current crisis of COVID-19. The Mass vaccination first started in the UK on 8th December 2020 and soon afterward covered all of the globe. Now sufficient data are available to analyse and compare some results to explore many aftereffects of vaccination. Some influence variables on transmissions of the disease were discussed e.g., mass vaccination, lockdown and seasonality. To address seasonality, similarities between COVID-19 and seasonal Flu are discussed to gain useful insight. Like Flu, seasonality was shown to play a dominant role in transmissions of COVID-19 in the Eu-rope and US. In terms of mass vaccination, adverse reactions after vaccination received attention, as health and safety issues of the general public are of prime importance. Apart from direct side effects, the secondary effect of mass vaccination needs attention too. After the initiation of vaccination programme, almost all countries experienced a sudden surge of transmission and most countries had to impose strict lockdown measures. Many countries, those showed a low prevalence of the disease, suddenly showed a steep jump after the onset of mass vaccination. Some countries even followed a synchronized pattern between the rate of transmissions and the variation of vaccine doses; the pattern seemed distinct with the sudden steep rise/fall in vaccine doses (e.g., countries India, Indonesia among others). In that context, fast mutation of the virus and new variants after mass vaccination and possible mechanisms/consequences were discussed.

Balanced discussion, critical and open analyses are desperately needed in the current crucial stage. Debating, questioning and criticism are always the foundation of good science and the main pillars to its progress. Following that objective, it is an effort to explore pragmatically, areas relating to the effectiveness of the COVID-19 vaccine and the exit strategy via the pathway of vaccination. Policymakers, academics, patients and common people will be greatly benefitted from such critical, transparent and balanced analyses.
\end{abstract}

\section{Hosted file}

preprints-54155-presentation.pdf available at https://authorea.com/users/336178/articles/ 552708-exit-strategy-of-covid-19-crisis-and-pathways-of-vaccination 